\title{
Aerodynamic Damping Analysis of a Vane-type Multi-Function Air Data Probe
}

\author{
Yung-Gyo Lee* and Young-Min Park** \\ Korea Aerospace Research Institute, Daejeon 305-806, Korea.
}

\begin{abstract}
Configuration design, analysis, and wind tunnel test of a vane-type multi-function air data probe (MFP) was described. First, numerical analysis was conducted for the initial configuration of the MFP in order to investigate aerodynamic characteristics. Then, the design was modified to improve static and dynamic stability for better response characteristics. The modified configuration design was verified through wind tunnel tests. The test results are also used to verify the accuracy of the analytical method. The analytically estimated aerodynamic damping provided by the Navier-Stokes equation solver correlated well with the wind tunnel test results. According to the calculation, the damping coefficient estimated from ramp motion analysis yielded a better correlation with the wind tunnel test than pitch oscillation analysis.
\end{abstract}

Key words: multi-function air data probe, damping, CFD, wind tunnel test

\section{Introduction}

The traditional air data system of an aircraft measures the airspeed and the altitude by using Pitot-static probe and also measures the angle of attack by using the vane type angle of attack sensor independently. The separated measurement system requires very complex pneumatic lines and wires to connect equipments and occupies much space for installation. On the contrary, the MFP measures total pressure, static pressure and angle of attack simultaneously within a single air data sensor unit ${ }^{1}$.

Typical MFPs have an embedded data processor unit located below the probe for direct processing of sensor information. Therefore, the MFP can measure airspeed, altitude and angle of attack simultaneously and can transmit the digital signal of data to the flight control computer directly. This kind of integrated concept makes the air data system very simple and also reduces its weight and volume as shown in Fig. 1. The simplified air data system has the advantage of minimizing the effort required for installation, operation, and maintenance ${ }^{2}$. Following the trend of the simple and integrated air data system, the present state-of- the-art aircrafts are being equipped with multi-function type air data sensor more and more, for example, the A-380 as a commercial aircraft and F-22 as a military aircraft. Recently, various kinds of the MFP configurations are in development and commercially available from companies such as Goodrich $^{2}$, Thales ${ }^{3}$, and Aerosonic ${ }^{4}$ in Fig. 2.

MFP can be separated into two categories, the multi-hole Pitot-static probe type and rotating vane type. Of the two types, a vane-type MFP has total and static pressure holes combined with a rotating vane for the measurement of angle

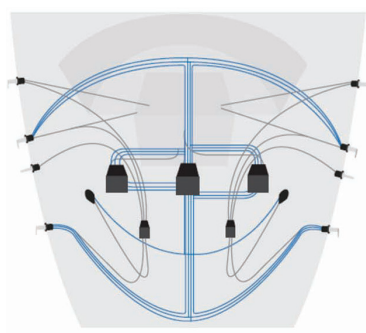

(a) Traditional air data system

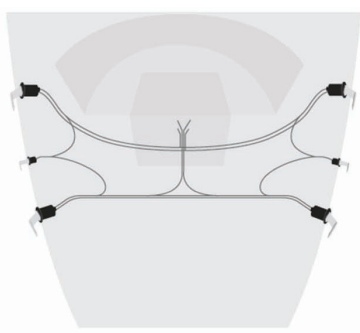

(b) MFP(SmartProbe $\left.{ }^{\mathrm{TM}}\right)$ air data system.
Fig. 1. Comparison of traditional and MFP air data system
This is an Open Access article distributed under the terms of the Creative Commons Attribution Non-Commercial License (http://creativecommons.org/licenses/by$\mathrm{nc} / 3.0 /$ which permits unrestricted non-commercial use, distribution, and reproduction in any medium, provided the original work is properly cited. (c) * Principal Research Engineer

** Senior Research Engineer, Corresponding author : ympark@kari.re.kr 


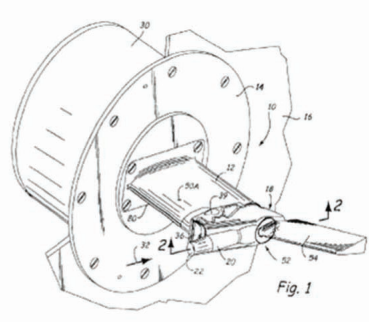

(a) Goodrich

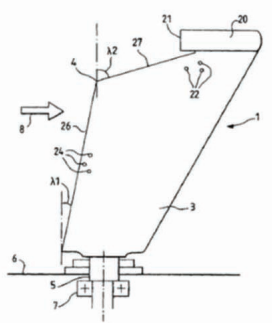

b) Thales

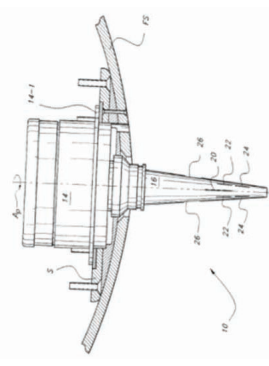

c) Aerosonic

Fig. 2. Rotating Vane-type Multi-function Probes

of attack. A vane-type MFP always guarantees the accuracy because the vane of the MFP automatically aligns itself with any flow direction and this mechanism minimizes the pressure measuring error under various flight conditions. The disadvantages of the vane-type MFP are well known as the response time delay and the vibration from overshoot because heavy and light inertia of the vane prohibit the alignment of vane along the freestream. In order to avoid such disadvantages, the shape of vane or the mechanical damper should be designed elaborately in the design process.

In the present paper, the aerodynamic attributes of the baseline model are understood through computational fluid dynamics (CFD) analysis, the design is modified to improve static and dynamic stability and obtain better response characteristics. A vane-type MFP from Aerosonic is selected as the baseline configuration. This model has been used for the previous researches in regard to the aerodynamic stiffness and damping analysis of the vane-type probe by the authors $^{5}$. The dynamic response time requirement for the MFP is defined as a time constant, and it should be no greater than $100 \mathrm{msec}$ at a speed of $120 \mathrm{kts}$ as typical requirements ${ }^{6}$.

\section{Initial Configuration and Analysis of Aero- dynamic Characteristics}

The initial configuration of the MFP from Aerosonic is shown in Fig. 3. Designed to be applied to state-of-the-art

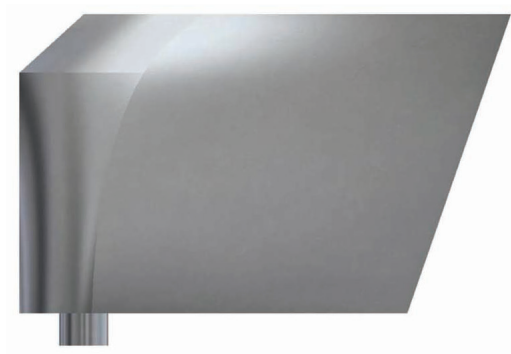

fighter aircrafts, this MFP takes into account a heating sheet for anti-icing and de-icing. However, since the aerodynamic damping of this baseline configuration is not satisfactory, the system needs an additional mechanical damper. As the configuration update presented in this study improves the aerodynamic damping, the mechanical damper is expected to be reduced in size, yielding less system weight and cost.

\subsection{Aerodynamic Stiffness}

In order to obtain the aerodynamic stiffness, a NavierStokes equation solver was used for the analysis along with the Spalart-Allmaras turbulence model. The grid is generated to have 15 viscous boundary layers on the surface, where y+ is less than 10. The total number of nodes is $1,500,000$. The physical grid for this analysis is shown in Fig. 4. The CFD analysis is conducted for a flow speed of $120 \mathrm{kts}$, where the

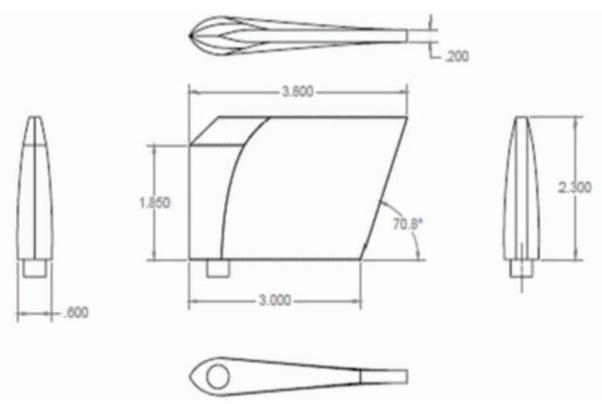

Fig. 3. 3-view of the initial MFP configuration

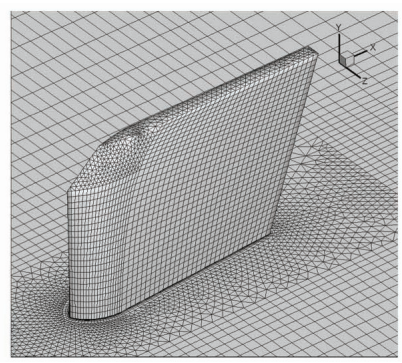

Fig. 4. Grid of the initial MFP configuration 
angle of attack is within the linear bounds of -5 deg to $5 \mathrm{deg}$. Figure 5 presents the aerodynamic stiffness for various Mach conditions. The representative aerodynamic stiffness of initial configuration is $0.23 \mathrm{Nm} / \mathrm{rad}$. The design requirement of aerodynamic stiffness is to increase by $100 \%$.

\subsection{Aerodynamic Damping}

The aerodynamic damping of an aircraft can be estimated using empirical formulas, such as Datcom, or measured through dynamic stability wind tunnel tests using a rotary balance $^{7}$. In this study, however, it is calculated using a CFD tool. The damping coefficient can be calculated based on two motions: pitch oscillation and ramp motion ${ }^{8}$. A brief introduction to the analysis methods using these motions is given below.

\section{- Pitch oscillation}

$$
\begin{aligned}
& \text { Motion: } \theta=\theta_{o}+\theta_{a m p} \sin (\omega t) \\
& \dot{\theta}(0)=\dot{\theta}_{o}, \ddot{\theta}(0)=0 \\
& \text { Damping : } \gamma_{a}(0)=\tau(0) / \dot{\theta}_{o}
\end{aligned}
$$

The vane oscillates in harmonic motion around the nominal angle of attack, or pitch angle, $\theta_{o}$ with constant amplitude $\theta_{a m p}$. The damping is calculated at the nominal angle $\theta_{o}$, at which the angular acceleration becomes zero.

\section{- Ramp motion (pitch-up with constant $\dot{\theta}$ )}

$$
\text { Damping: }\left(\tau_{\text {dynam } \dot{\varepsilon}}(\theta)-\tau_{\text {static }}(\theta)\right) / \dot{\theta}
$$

If the vane pitches at a constant rate, the change in moment from the static moment can be calculated. Dividing the moment change by the angular speed yields the damping coefficient. This method is efficient as it requires only one calculation to obtain all the damping coefficients within the angle of attack bounds.

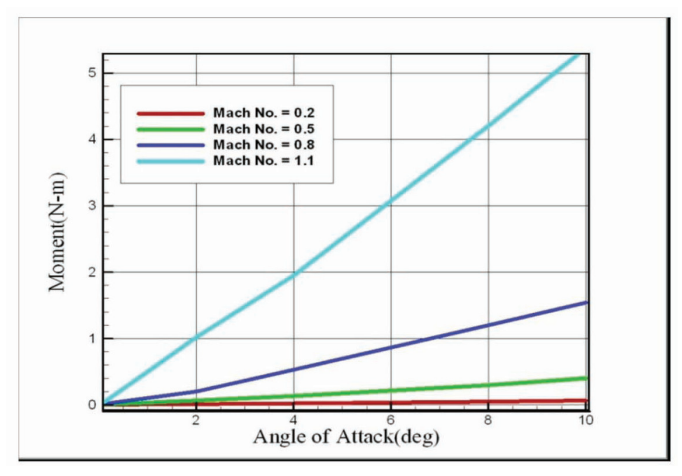

Fig. 5. Aerodynamic stiffness of initial MFP.
The pitch oscillation method that uses dual time stepping in its calculations gives no single number for the result as shown in Fig. 6. Instead, the result varies depending on the time step and the number of sub-iterations. This is probably because the present flow solver restricts the dynamic mesh to first order temporal accuracy. On the other hand, second order time accuracy is available when using the ramp motion method that incorporates the rotating mesh technique. Therefore, the damping coefficient from ramp motion analysis was used for the analysis of dynamic response. The damping coefficient of the initial configuration estimated from this method is $0.0012 \mathrm{Nmsec} / \mathrm{rad}$ at a wind speed of 120 kts. The design requirement of aerodynamic damping is to increase by $30 \%$.

\subsection{Dynamic Response}

The oscillating MFP is an initial value problem (IVP) and can be simplified by a second-order ordinary differential equation as follows

$$
\begin{array}{lr}
J \ddot{\theta}+\gamma(\theta) \dot{\theta}+k(\theta) \theta=k_{a} \alpha-\tau_{d} \operatorname{sgn}(\dot{\theta}) & \mathrm{t}>0 \\
\theta=\theta_{o}, \dot{\theta}=0 & \mathrm{t}=0
\end{array}
$$

Where $\mathrm{J}$ is the polar moment of inertia, $\gamma$ is the damping coefficient, $\mathrm{k}$ is the stiffness, and $\tau_{\mathrm{d}}$ is the friction coefficient of the bearing. J is calculated using Solid Works, and only the aerodynamic damping is included. It is assumed that there is no mechanical damper.

\section{Configuration}

A new configuration is designed that improves the static stiffness and the aerodynamic damping over those in the initial MFP. The new MFP has better accuracy in measuring the pressure and angle of attack. In addition, it minimizes the use of the mechanical damper, which may ultimately reduce system cost and weight.

\subsection{Design Method}

The direction of the design modification is determined by estimating how much improvement in aerodynamic damping can be achieved by adding a surface to the initial MFP. The additional surface has an area of A2 and a center of pressure at a distance D2 from the rotation axis. It is attached to the baseline, which has an area Al and a center of pressure at a distance D1 from the same axis. In order to simplify the governing equation, the following assumptions are established:

- The center of pitch rotation is located before the probe 
- The center of pressure is fixed regardless of the angle of attack

As Fig. 7. illustrates, when the probe rotates with an angular speed $\omega$, the upwash component occurs on the downstream side of the model. This upwash increases the angle of attack, and thus the aerodynamic damping, causing a moment larger than the static $(\omega=0)$ condition. Here, the increment of the moment from the static condition is

$$
\frac{\Delta C_{m}}{\omega}=-D^{2} k \rho \frac{v S}{2} \approx C_{m q}
$$

This also represents the amount of the aerodynamic damping.

It is noticeable from the equation above that $\mathrm{C}_{m q}$, is negative (i.e. the system has positive damping) when $\kappa$ is positive. This creates a dynamically stable condition as shown in Fig. 8. The slope of pitching moment, which is defined to be the opposite sign of aerodynamic stiffness by sign convention, $\mathrm{C}_{\mathrm{m} \alpha}$, also becomes negative when $\mathrm{K}$ is positive, meaning the system is statistically stable as well. As the angle of attack becomes excessively large and the flow begins to stall, $k$ turns negative and the system becomes unstable.

The damping increment for adding area $\mathrm{A} 2$ at the distance $\mathrm{D} 2$ is determined as

$$
\frac{\tau_{2}}{\tau_{1}}=\left(\frac{D_{2}}{D_{1}}\right)^{2}\left(\frac{A_{2}}{A_{1}}\right)
$$

For example, adding A2, which is $20 \%$ of $\mathrm{A} 1$, at D2, which is two times D1, increases the aerodynamic damping by $80 \%$.

\subsection{Modified Configurations}

Design 1 has almost the same area as the initial configuration. However, it is designed to have a greater area at the location farthest from the axis of rotation. In addition, the brushing area against the wall is minimized so that the MFP can be installed regardless of the surface condition of

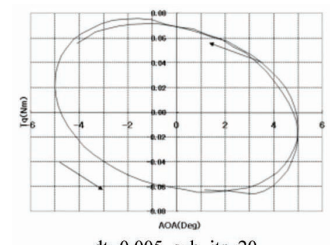

$\mathrm{dt}=0.005$, sub_itr $=20$

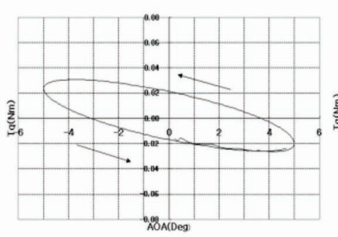

$\mathrm{dt}=0.001$, sub_itr $=20$

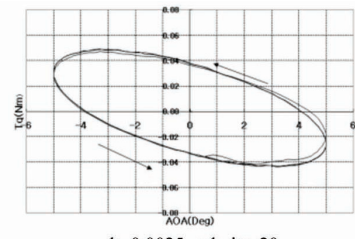

$\mathrm{dt}=0.0025$, sub_itr $=20$

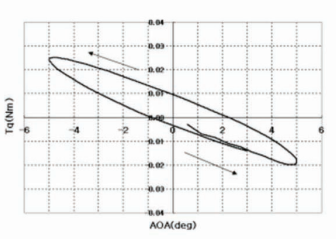

$\mathrm{dt}=0.005$, sub_itr $=50$ (a) Pitch oscillation the aircraft. As the MFP is installed apart from the aircraft surface, the need for anti-icing or de-icing of the surface shrinks. Therefore, the overall system becomes simpler.

On the other hand, Design 2 maximizes aerodynamic damping, especially for an aircraft surface for which antiicing or de-icing is available.

\section{Wind Tunnel Test}

\subsection{Test Facility}

The static and dynamic response tests are conducted in Aerosonic's subsonic wind tunnel. The specifications of this wind tunnel are very suitable for this particular test: it is a suction type wind tunnel with a maximum wind speed of 160 kts and a flow angularity of less than $0.2 \mathrm{deg}$.

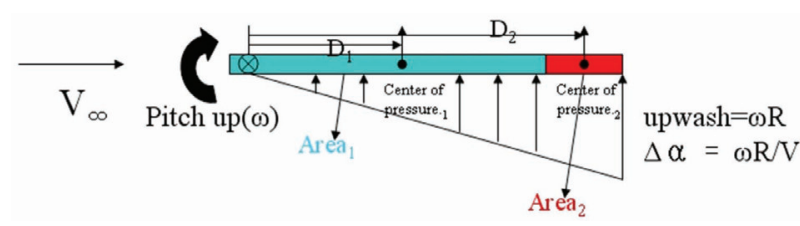

Fig. 7. Probe in pitching motion

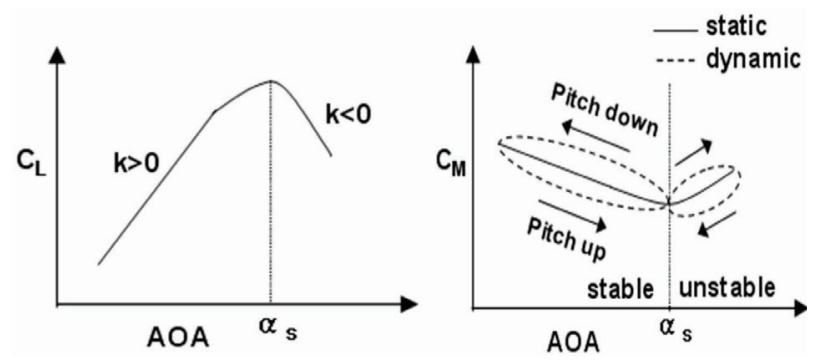

Fig. 8. The aerodynamic stiffness effect on the stability.

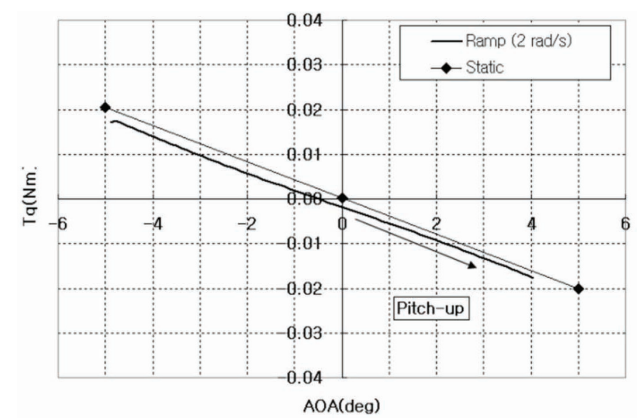

(b) Ramp motion

Fig. 6. Unsteady aerodynamic simulation results for damping analysis. 
Figure 9 shows that the test rig consists of the MFP model, pressure sensors from Memscap, location sensors from Netzer, a counter mass to balance the weight, and the data acquisition system.

\subsection{Multi-function Probe Model}

The wind tunnel models of the MFP are fabricated using stereolithography (SLA) for rapid construction. The polar moment of inertia of the SLA model is matched with that of the actual aluminum MFP by inserting a brass plate into the model. The fabricated MFP wind tunnel test models are shown in Fig. 10.

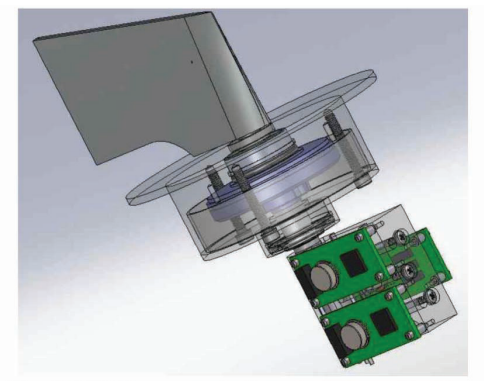

Fig. 9. MFP sensor rig for wind tunnel testing

(Vane, Netzer encoder, 3 Memscap pressure sensors)

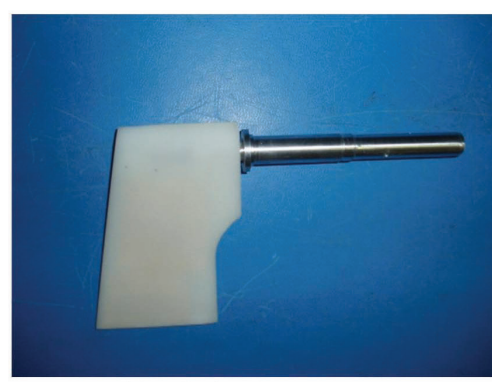

(a) Design 1

Fig. 10. Wind tunnel test model of design 1 and design 2

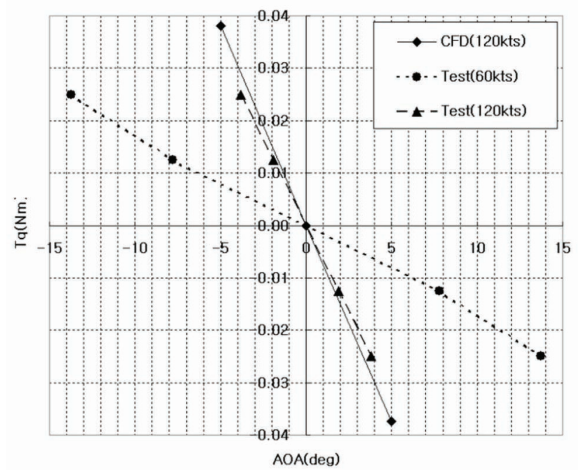

(a) Design 1

\section{Discussion of New Design}

The results from the CFD analysis are compared with results from the wind tunnel test for the new MFP designs. The aerodynamic stiffness is measured in the test by recording the displacement angle corresponding to the moment applied to the model. The moment is adjusted by adding mass to the test rig. The results from the calculation and tests are shown in Fig. 11. Aerodynamic stiffness is defined here to be the opposite sign of the moment curve slope. It is noticeable from the figure that the calculations correlate well with the wind tunnel tests for $120 \mathrm{kts}$ cases, although the test result of Design 2 is $21 \%$ lower than that of analysis. The summary of the aerodynamic stiffness of designs 1 and 2 is given in Table 1 .

The aerodynamic damping from the test is estimated using the dynamic response data shown in Fig. 12. Using the dynamic response equation, the damping value is found by shooting until the response matches the time history from the test. In the course of determining the damping, the polar moment of inertia J, one of the important parameters, is calculated using Solid Works. The aerodynamic stiffness obtained from previous test is used, and the internal stiffness and bearing friction coefficient are separately measured in

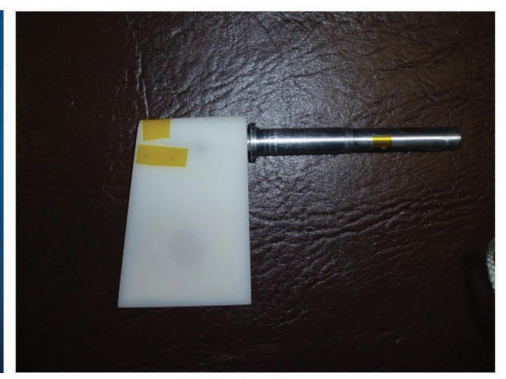

(b) design 2

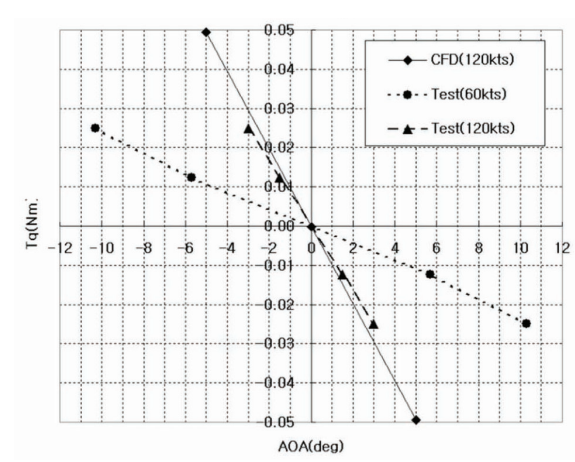

(b) design 2

Fig. 11. Pitching moment of design 1 and design2(120kts) 
advance.

The summary for the aerodynamic damping of designs 1 and 2 is given in Table 2, showing the test result of Design 2 is $19 \%$ lower than that of analysis.

According to the CFD analysis, separation is delayed at the rear part of the vane, resulting in overestimation of aerodynamic stiffness. Furthermore, high aerodynamic stiffness, which means more pitching moment, essentially results in high aerodynamic damping, according to eqn. (6).

\section{Conclusions}

In this study, modified MFPs were designed and tested. It was verified by analysis and testing that they have better aerodynamic performance than the initial MFP. Analysis showed that the new MFPs result is more than two times greater in static stiffness and 33\% higher in aerodynamic damping than the baseline configuration. In addition, it is verified that the numerical estimations of aerodynamic stiffness and damping using the CFD solver, especially for ramp motion, correlate well with the test results.

\section{References}

[1] Goodrich (2011), Sensor and Integrated Systems, URL

Table 1. Aerodynamic stiffness coefficient of designs 1 and 2

\begin{tabular}{cccc}
\hline \multirow{2}{*}{ Model } & \multirow{2}{*}{$\begin{array}{c}\text { Wind } \\
\text { Speed }\end{array}$} & \multicolumn{2}{c}{ Stiffness $(\mathrm{Nm} / \mathrm{rad})$} \\
\cline { 3 - 4 } & $(\mathbf{k t s})$ & Experimental & Analytical \\
\hline \multirow{2}{*}{ Design 1 } & 60 & 0.098 & \\
\cline { 2 - 4 } & 120 & 0.39 & 0.43 \\
\hline \multirow{2}{*}{ Design 2 } & 60 & 0.12 & \\
\cline { 2 - 4 } & 120 & 0.45 & 0.57 \\
\hline
\end{tabular}

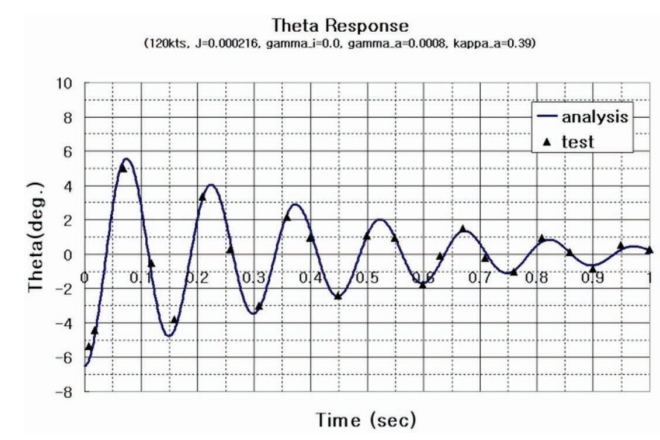

(a) Design 1

Fig. 12. Dynamic response of design 1 and design2(120kts)
: http://www.goodrich.com/Goodrich/Businesses/Sensorsand-Interated-Systems (accessed Aug 20 2012).

[2] Greg, A., Dennis, J., John, H., Mark, R., James, A., John, R., and Dana, A., "Multi-Function Air Data Sensing Probe Having an Angle of Attack Vane," US Patent No. 6941805B2, 2003.

[3] Lionel, C., and Nikolas, H., "Multi-Function Probe with Variable Flexure," US Patent No. 7155968B2, 2007.

[4] Margaret, A., Guntis, U., and David, N., "Multi-Function Aircraft Probes,” US Patent No. 6012331, 2000.

[5] Lee, Y., Park, Y., Ahn, S., Yeom, C., and Lee, D., “Design and Wind Tunnel Tests of a Multi-Function Air Data Probe," Proceedings of the 2010 Spring Korean Society for Aeronautical and Space Sciences, Pyungchang, Korea, 2010.

[6] Park, Y., Choi, I., Lee, Y., Kwon, K., Kim, S., and Hwang I., "Aerodynamic Design of a Multi-function Air Data Sensor by Using CFD and Wind Tunnel Test," Journal of Korean Society of Computational Fluid Engineering, Vol. 15, No. 3, 2010, pp. 32-38.

[7] Chang. B., Ok. H., and Lee. Y., "Forced Oscillation Wind Tunnel Test of a 50m Airship," Journal of the Korean Society for Aeronautical and Space Sciences, Vol. 31, No. 6, 2003, pp. 17-22.

[8] Lee. Y., and Lan. C. E., "Navier-Stokes Analysis of Tunnel Wall Interference Effects on Pitching Delta Wings," Journal of Aircraft, Vol. 41, No. 3, 2004, pp. 547-554.

Table 2. Aerodynamic damping coefficient of designs 1 and 2

\begin{tabular}{cccc}
\hline \multirow{2}{*}{ Model } & \multicolumn{2}{c}{$\begin{array}{c}\text { Wind } \\
\text { Speed }\end{array}$} & \multicolumn{2}{c}{ Damping (Nmsec/rad) } \\
\cline { 2 - 4 } & (kts) & Experimental & Analytical \\
\hline \multirow{2}{*}{ Design 1 } & 60 & 0.004 & \\
\cline { 2 - 4 } & 120 & 0.0008 & 0.0007 \\
\hline \multirow{2}{*}{ Design 2 } & 60 & 0.0007 & \\
\cline { 2 - 4 } & 120 & 0.0013 & 0.0016 \\
\hline
\end{tabular}

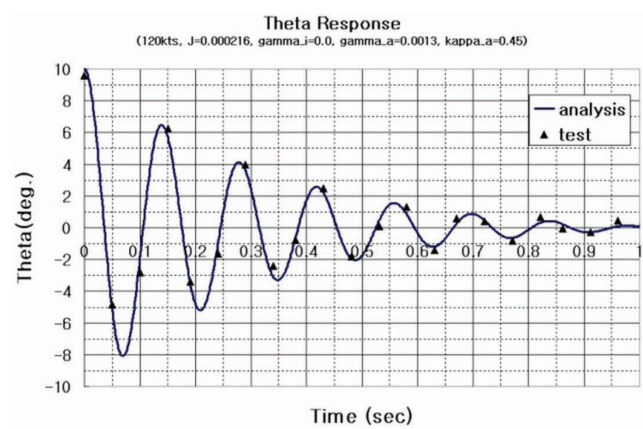

(b) design 2 